\title{
Los bancos de lberoamérica a través de las visiones y misiones corporativas
}

Guillermo Murillo-Vargas*, Carlos H. González-Campo y Steven A. Piñero-Buritica

Universidad del Valle, Sede San Fernando, Cali-Colombia (correo-e: guillermo.murillo@correounivalle.edu.co; carlosh.gonzalez@correounivalle.edu.co; steven.pineros@correounivalle.edu.co)

* Autor a quien debe ser dirigida la correspondencia.

Recibido Sep. 3, 2020; Aceptado Oct. 29, 2020; Versión final Dic. 16, 2020, Publicado Abr. 2021

\begin{abstract}
Resumen
El objetivo de este estudio es analizar la banca de lberoamérica a través de las misiones y las visiones corporativas, con el fin de reconocer cuáles son sus principales prioridades estratégicas como organizaciones. La muestra de esta investigación cualitativa consiste en 39 bancos de la región lberoamericana que han sido considerados como los principales bancos de sus países. Las misiones y las visiones fueron obtenidas de las páginas web de cada banco y se analizaron desde diferentes perspectivas teóricas. Los resultados revelan la prioridad que los bancos lberoamericanos le dan al desarrollo económico de los países donde se encuentra y su enfoque total hacía los clientes, donde centran todos sus procesos. Se concluye que la banca en lberoamérica tiene dentro de sus prioridades, vistos a través de las misiones y visiones organizacionales, el desarrollo económico dentro de su enfoque corporativo, centrado en las necesidades de sus clientes en la banca personal y corporativa.
\end{abstract}

Palabras clave: visión; misión; bancos; Iberoamérica; planeación estratégica

\section{Ibero-American banks through corporate visions and missions}

\begin{abstract}
The present research study aims to determine the main strategic priorities of Ibero-American banks by analyzing their missions and corporate visions. The sample of this qualitative research study consists of 39 Ibero-American banks that are considered the most important banks in their respective countries. The missions and visions for each bank were obtained from their official websites. The results reveal that Ibero-American banks prioritize the economic development of their respective locations and that the totality of their focus is on their customers, centralizing all processes on them. It is concluded that among Ibero-American banks' priorities are, as seen through corporate visions and missions, economic development as their corporate aim, focused on the needs of their individual and corporate customers.
\end{abstract}

Keywords: vision; mission; banks; Ibero-America; strategic planning 


\section{INTRODUCCIÓN}

La banca internacional ha estado presente en la vida social y económica durante mucho tiempo, es la transformadora del dinero de los personas y empresas, permitiendo aumentar la riqueza de las naciones dada la capacidad que tiene de invertir el dinero que capta (Weber, 1994). Además, es uno de los sectores que más recursos aporta a la economía mundial, es un conjunto de diferentes instituciones que brindan seguridad al movimiento del dinero y a los diferentes medios de pago. Los bancos garantizan que la asignación de estos recursos se realice eficientemente, contribuyen al desarrollo y la estabilidad de las regiones, fomentando la inversión y el ahorro (Fang et al., 2014). De esta forma, a lo largo de su historia la banca ha enfrentado diferentes desafíos, como, por ejemplo, la crisis a finales de los años noventa en diferentes países del primer mundo y que impacto la economía global, por actos de corrupción, crímenes en el sistema financiero, entre otros acontecimientos (Schure et al., 2004).

De la misma forma, un hecho relevante fue la crisis económica internacional de 2008, que llevó a la quiebra y posterior salvamento de los bancos más grandes del mundo, producto de la crisis de hipotecas suprime (Ting, 2017). Este suceso, dio paso a cambios en la regulación financiera, como también cambios estructurales en el mercado bancario y financiero (Segura y Valls, 2018). De esta forma, aparece en la planeación estratégica un aspecto fundamental que son las declaraciones de la misión y la visión y que cumplen un papel importante como fuente de inspiración hacia la consecución de los objetivos (Braun et al., 2012; Ingenhoff y Fuhrer, 2010). Además, según Billington (1996), la misión y la visión ayudan a entender el propósito social de cada una y también explican los propósitos de las organizaciones de manera concisa, siempre y cuando declare objetivos alcanzables.

Dada la importancia que tiene el sector bancario para la economía en todos los países y para las personas es importante entender ¿Cuáles son estos objetivos? Además, de reconocer ¿Cuáles son sus prioridades corporativas? Por lo tanto, una forma de hacerlo es mediante el análisis cualitativo de estas declaraciones de Misión y Visión. Es de esta forma, que resulta importante estudiar la banca en Iberoamérica, una región clave, con amplia inversión extranjera directa, una banca internacionalizada y países industrializados (Casilda, 1999). España por su parte, cuenta con una estructura bancaria solida con presencia en diferentes países de Iberoamérica y en otras partes del mundo. Según la Revista The Banker en el 2020, en la posición 15 y 33 a nivel mundial de los mejores bancos están Santander y BBVA respectivamente. Por su parte, en Latinoamérica las economías más fuertes y con los bancos con mayores ganancias son Brasil y México (Caplen, 2020). Los resultados de este estudio van encaminados a la importancia que tiene el cliente en sus misiones y visiones, puesto que es la palabra que más frecuencia tiene junto a otros grupos de interés. De esta misma forma, se evidencia el compromiso que tienen los bancos con el desarrollo de las regiones donde tienen presencia en proyectos junto a múltiples instituciones estatales.

\section{OTROS ANTECEDENTES}

Hay dos aspectos que necesitan ser revisados y analizados para este trabajo: importancia de la misión y la visión en la administración y las prioridades de la banca frente al cliente

\section{Importancia de la misión y la visión}

La declaración de la misiones y visión es considerada por algunos autores como una de las piedras angulares en la planeación estratégica en la administración de cualquier organización. Es de esta forma que, ya sean organizaciones Públicas o privadas, con ánimo o sin ánimo de lucro, multinacionales, grandes empresas y Pyme. Por lo cual, una declaración de Misión y Visión distinguirá a las empresas entre sus competidores, mostrando en sus declaraciones sus características únicas frente a las demás (Allen et al., 2018). Adicionalmente, estas son una orientación en el proceso de toma de decisiones, para la consecución de metas y muestra los mecanismos que permiten establecer relaciones con los stakeholders (Freeman et al., 2010). El propósito final de cualquier organización es alinear sus valores con las expectativas de los grupos de interés, mientras se pregunta ¿cuál es nuestro negocio? y ¿Cuál es el objetivo de nuestro negocio?, la declaración de la misión muestra la razón por la cual existe una empresa y evidencia su orientación estratégica en el sector que se encuentra (Freeman et al., 2010).

La formulación de la Visión y Misión es la etapa más crucial en la planeación estratégica y por esto se deben identificar y declarar correctamente, puesto que le agrega a la organización una identidad y fidelidad tanto con los clientes, como en los colaboradores, creando la guía de todas las actividades dentro de las organizaciones (Allen et al., 2018). No obstante, en algunas ocasiones cuando no se declara o no se apropia adecuadamente o no se entiende la diferencia entre la misión y la visión, tiene una afectación dentro de los empleados de la organización al no estar alineados con los objetivos de esta, algo que también le puede suceder a los clientes al no entender correctamente la Misión y Visión (Papulova, 2014). Además, dada la importancia que tienen estas declaraciones es importante que al realizar tengan un contenido de calidad (Ahmed et al., 2017; Allen et al., 2018). 
La declaración de una misión debe describir cómo se logrará lo que la empresa quiere llegar a hacer. Aquí se expresan los objetivos, relacionando los diferentes grupos de interés y los valores corporativos. Generalmente, habla del presente, pero enfrentando los desafíos del futuro. Lo anterior, sive para establecer metas dentro de la organización y los diferentes grupos de interés tendrán una mejor comprensión de la empresa. Además, la misión puede cambiar, pero siempre debe tener en cuenta los valores de la empresa, el cliente y las necesidades de esta (Brickson, 2007). Adicionalmente, como lo expone Melewar (2003), es una fuente de liderazgo, muestra los valores, las metas de la organización y la forma en que se integran todas las partes interesadas en ella. De acuerdo Ahmed et al. (2017), la misión tiene 3 beneficios para las empresas: Guía la dirección, crea una imagen positiva de la empresa y es una estrategia de mercado adentro como afuera de la organización.

La visión se centra en lo que la organización desea llegar a convertirse. los valores y los propósitos de los negocios deben ser expresado, respondiendo la pregunta ¿Dónde queremos estar? Por lo tanto, la visión se centra en el futuro, es una panorámica de como espera proyectarse la organización en los siguientes años desde ahora, lo que motiva a las empresas para dar lo mejor. Además, provee de sentido la existencia de la organización. La visión puede ir cambiando a medida que se va transformando la empresa, pero claramente es mucho más estable que la misión. Siempre debe ser concreta, clara, sin paso a las interpretaciones y siempre se escribe dando un mensaje de un futuro promisorio (Ingenhoff y Fuhrer, 2010).

La mayoría de los bancos cuenta con una misión y visión establecidas y usan estas declaraciones como un modo de expresar el carácter corporativo de la empresa, estas se encuentran reflejadas en las mayorías de los documentos institucionales o plataformas virtuales, entre ellas las webs de cada entidad. En los años recientes, han surgido temas como la responsabilidad social corporativa dados los grandes fracasos de las compañías financieras, esto ha generado impactos negativos en el desarrollo económico de los países y ha cambiado las estrategias de las corporaciones (Ahmed et al., 2017). Lo anterior, demanda un compromiso con los diferentes grupos de internet y estas estrategias de planeación, pretender conectar a las empresas con sus valores, teniendo en cuanto su relación con los stakeholders y el medio ambiente (Platonova et al., 2018).

\section{Prioridades de la Banca}

Actualmente la banca se encuentra enfocado en el sector de la tecnología y la experiencia hacia el cliente, ofreciéndole gran variedad de servicios. Es así, que la banca móvil o las Fintech se vuelve un punto importante para las entidades financieras. Es por esto, que resurge un nuevo tipo de gobierno bancario o de responsabilidad social corporativa donde se pretende poner al cliente en el centro de toda la operación, lo que conlleva a un cambio importante y se crean nuevos escenarios donde estas entidades deben adaptarse a las exigencias de los nuevos y viejos consumidores (Platonova et al., 2018).

Posterior a la recesión económica mundial del 2008, la confianza del consumidor bancario se deterioró dada las prácticas tradicionales, el uso de información poco clara, burocracia excesiva, el distanciamiento con el cliente y sus necesidades dejaban en grandes problemas al sector bancario (Ahmed et al., 2017; Platonova et al., 2018). En estudios como Cotta (2011), se evidencia la estrategia de los bancos para construir confianza con los clientes, como uno de sus propósitos principales. Es por esto, que los bancos, en sus diferentes informes de responsabilidad corporativa, recalcan el compromiso que tienen estos con el desarrollo económico y social, los bancos más grandes quieren seguir mejorando su rendimiento y acelerar el proceso de digitalización para ofrecer productos más innovadores y mejorar las experiencias con los clientes. De esta forma, los bancos se tienen como objetivo mejorar las prácticas de responsabilidad y sostenibilidad, maximizar la creación de valor para los grupos de interés creando relaciones de largo plazo basadas en la confianza y trasparencia (Segura y Valls, 2018).

Asimismo, la seguridad de la información financiera se ha vuelto en un objetivo primordial para poder implementar una transformación digital eficiente, puesto que algunos de clientes aún desconfían de este tipo de tecnologías. También, entre sus prioridades esta la retención de personal con alta formación, lo que resulta fundamental para avanzar en este proceso tecnológico. Todo lo anterior, tiene como finalidad mejorar los procesos internos de los bancos y de esa forma incrementar la eficiencia en la gestión del riesgo, en el cumplimiento de objetivos, la optimización de balances, entre otras necesidades (Ahmed et al., 2017; Platonova et al., 2018; Segura y Valls, 2018).

En la revisión de literatura es evidente que el campo de estudio de las orientaciones estratégicas de los bancos desde el análisis de sus misiones y visiones, es poco explorando, en las búsquedas en Scopus y Web of Sciencie, con diferentes ecuaciones de búsqueda, los resultados fueron pocos, entre los que se encuentra la utilización de métodos cualitativos en estudios como Ahmed (2010); Bakoğlu y Aşkun (2007); Bartkus et al. (2006), Tan et al. (2017) o Kontolaimou et al. (2006), son menos los estudios donde se usan métodos cuantitativos, entre ellos, Dhananjay y Suresh (2015), y con métodos mixtos, se encuentran Platonova et al. (2018) y Ahmed (2016). En ninguna de las anteriores publicaciones el objeto de estudios fueron bancos de 
Iberoamérica, se observa un reciente interés por analizar sobre los bancos islámicos y los bancos en la india, y en otros casos más tradicionales como los bancos de Estados unidos y bancos europeos. Desde esta perspectiva se evidencia el aporte del presente artículo.

\section{METODOLOGÍA}

En una fase previa al análisis cualitativo de las misiones y de las visiones de los Bancos en lberoamérica, se realiza una revisión bibliografía en Scopus y Web of Science, para indagar por el estado del arte de estudios similares, en la misma región, o en otras regiones. Esta revisión permitió identificar que es un campo de conocimiento poco estudiado, donde no hay referencia previa de estudios idénticos en lberoamérica, y tampoco se evidenciaron diferencias con estudios en otras regiones, estas diferencias van desde el método de investigación hasta algunos hallazgos, que son contrastados en la sección de discusión.

El estudio se realizó mediante un análisis descriptivo con un enfoque cualitativo utilizando los datos de la información obtenida de los documentos oficiales y de las páginas web de los 39 bancos de 13 países de Iberoamérica escogidos de acuerdo con diferentes rankings de forma estructural, como se observa en la Tabla 1.

Tabla 1. Bancos seleccionados para el estudio.

\begin{tabular}{|c|c|c|c|c|c|}
\hline$N^{\circ}$ & País & Nombre del Banco & Dirección web & Tipo de Propiedad & $\begin{array}{l}\text { Actualización } \\
\text { del sitio }\end{array}$ \\
\hline 1 & Argentina & Nación & www.bna.com.ar & Nacional-Estatal & $02 / 02 / 2020$ \\
\hline 2 & Argentina & Galicia & www.bancogalicia.com & Nacional-Privada & $21 / 10 / 2020$ \\
\hline 3 & Argentina & Santander Río & www.santander.com.ar & Extranjera-Privada & $06 / 05 / 2020$ \\
\hline 4 & Bolivia & Mercantilista Sta. Cruz & www.bmsc.com.bo & Nacional-Privada & $08 / 01 / 2020$ \\
\hline 5 & Bolivia & Unión & www.bancounion.com.bo & Nacional-Estatal & $21 / 11 / 2020$ \\
\hline 6 & Bolivia & Nacional de Bolivia & www.bnb.com.bo & Nacional-Privada & $27 / 01 / 2020$ \\
\hline 7 & Brasil & Itaú & www.itau.com.br & Nacional-Privada & $11 / 03 / 2020$ \\
\hline 8 & Brasil & do Brasil & www.bb.com.br & Nacional-Estatal & $06 / 01 / 2020$ \\
\hline 9 & Brasil & Bradesco & www.bradescori.com.br & Nacional-Privada & $31 / 10 / 2020$ \\
\hline 10 & Chile & $\mathrm{BCl}$ & www.bci.cl & Nacional-Privada & $04 / 09 / 2020$ \\
\hline 11 & Chile & Santander & www.santander.cl & Extranjera-Privada & $23 / 11 / 2020$ \\
\hline 12 & Chile & Estado & www.bancoestado.cl & Nacional-Estatal & $10 / 06 / 2020$ \\
\hline 13 & Colombia & Bancolombia & www.grupobancolombia.com & Nacional-Privada & $09 / 07 / 2020$ \\
\hline 14 & Colombia & de Bogotá & www.bancodebogota.com & Nacional-Privada & 10/07/2020 \\
\hline 15 & Colombia & Davivienda & ir.davivienda.com & Nacional-Privada & $01 / 04 / 2020$ \\
\hline 16 & Costa Rica & Nacional & www.bncr.fi.cr & Nacional-Estatal & $05 / 05 / 2020$ \\
\hline 17 & Costa Rica & de Costa Rica & www.bancobcr.com & Nacional-Estatal & $04 / 09 / 2020$ \\
\hline 18 & Costa Rica & San José & www.baccredomatic.com & Nacional-Privada & $17 / 11 / 2020$ \\
\hline 19 & Ecuador & Pichincha & www.pichincha.com & Nacional-Privada & 19/11/2020 \\
\hline 20 & Ecuador & Pacifico & www.bancodelpacifico.com & Nacional-Estatal & $02 / 03 / 2020$ \\
\hline 21 & Ecuador & Banco Guayaquil & www.bancoguayaquil.com & Nacional-Privada & $10 / 02 / 2020$ \\
\hline 22 & España & Santander & www.santander.com & Nacional-Privada & $22 / 04 / 2020$ \\
\hline 23 & España & BBVA & www.bbva.com & Nacional-Privada & $04 / 05 / 2020$ \\
\hline 24 & España & CaixaBank & www.caixabank.com & Nacional-Privada & 09/07/2020 \\
\hline 25 & México & BBVA México & www.bbva.mx & Extranjera-Privada & $09 / 11 / 2020$ \\
\hline 26 & México & Santander México & www.santander.com & Extranjera-Privada & $22 / 04 / 2020$ \\
\hline 27 & México & Banamex (Citibanamex) & www.banamex.com & Extranjera-Privada & $23 / 06 / 2019$ \\
\hline 28 & Panamá & General & www.bgeneral.com & Nacional-Privada & $02 / 03 / 2020$ \\
\hline 29 & Panamá & Nacional & www.banconal.com.pa & Nacional-Estatal & $01 / 05 / 2020$ \\
\hline 30 & Panamá & Banistmo & www.banistmo.com & Extranjera-Privada & $11 / 07 / 2020$ \\
\hline 31 & Paraguay & Continental & www.bancontinental.com.py & Nacional-Privada & $11 / 02 / 2020$ \\
\hline 32 & Paraguay & Itaú Paragua & www.itau.com.py & Extranjera-Privada & 17/05/2020 \\
\hline 33 & Paraguay & Regional & www.regional.com.py & Nacional-Privada & 22/09/2019 \\
\hline 34 & Perú & Crédito & www.viabcp.com & Nacional-Privada & $26 / 08 / 2020$ \\
\hline 35 & Perú & BBVA Perú & www.bbva.pe & Extranjera-Privada & $17 / 11 / 2020$ \\
\hline 36 & Perú & Scotiabank (Perú) & www.scotiabank.com.pe & Extranjera-Privada & $15 / 01 / 2020$ \\
\hline 37 & Portugal & Caixa Geral de Depósitos & www.cgd.pt & Nacional-Estatal & $22 / 07 / 2020$ \\
\hline 38 & Portugal & Millennium BCP & ind.millenniumbcp.pt & Nacional-Privado & $14 / 09 / 2020$ \\
\hline 39 & Portugal & Santander Totta & www.santander.pt & Extranjero-Privado & $22 / 05 / 2020$ \\
\hline
\end{tabular}


Consecutivamente, se seleccionaron los tres primeros bancos de cada país, en el caso de Latinoamérica se empleó el ranking de la Revista América Economía (Lacourt y Musalem, 2020), que muestra los 250 bancos que tuvieron los activos totales más altos en 2019. Por otra parte, España y Portugal no hacían parte de este clasificación, por lo cual, se identificaron otros rankings de alto reconocimiento, para el primero se utilizó el TOP 1000 de la revista The Banker en el 2020 y para el caso de Portugal el Top Banks in Portugal de ADV Ratings en 2020, firma canadiense que hace la escala de acuerdo a las utilidades anuales de cada banco. Inmediatamente, se procedió a visitar e identificar la misión y visión de cada uno de los bancos. Los anteriores datos se recolectaron del 1 al 15 de Julio del 2020. Adicionalmente, se verifico la fecha de actualización de todos los sitios web con el fin de constatar que la información era reciente.

La metodología de la investigación se realizó por medio de un análisis de contenido, ampliamente aceptado por la comunidad científica y en investigaciones de tipo cualitativo y descripto por su mecanismo de datos agregados, que permite analizar objetivamente la información (Vaismoradi et al., 2018). Por lo cual, el proceso de análisis de las misiones y las visiones se realizó por medio del programa ATLAS.ti óptimo para la investigación cualitativa de datos ver. 8.4. A continuación, al analizar las Misiones y Visiones, se pretendía ver cuáles eran las palabras que se repetían con mayor frecuencia, en primera instancia se procedió al análisis de lo general a lo específico, y posteriormente se realizado un análisis comparativo por tipo de estructura de propiedad, es decir, por públicas y privadas, tal como se puede observar en la Tabla 5 y 6 . De esta forma, para el análisis de los datos se seleccionaron las palabras que tuvieran una frecuencia mayor o igual a $10 \mathrm{y}$ las palabras que se relacionaban fueron unificadas para realizar un mejor estudio. Metodológicamente, en la visualización de las nubes de palabras en las Figuras 1 y 2 , el tamaño de la letra de cada palabra es proporcional con la frecuencia en el texto analizado.

Es importante resaltar que la fuente de las tablas y las gráficas presentes en este artículo fueron realizadas por medio de Atlas T.I por los autores, con la información obtenida de los diferentes sitios web de las páginas web, como las declaraciones de misiones y visiones en las mismas páginas, como también, los reportes, informes, entre otros documentos, que permitieron recopilar la información en una base de datos para su posteriormente procesamiento. Las páginas donde se tuvo dicha información se pueden ver en la Tabla 1.

\section{RESULTADOS}

En la Tabla 2, se presenta un panorama general de las palabras que más se repiten en las Misiones y Visiones, en el conjunto de los bancos que hacen parte de la investigación. En la Tabla 3, se presentan el resultado del análisis de las palabras con mayor frecuencia, en las misiones de los bancos en lberoamérica. En la Tabla 4, se presentan el resultado del análisis de las palabras con mayor frecuencia, en las visiones de los bancos en Iberoamérica.

Tabla 2. Frecuencia de palabras general entre las Misiones y Visiones de los bancos

\begin{tabular}{|l|c|c|}
\hline \multicolumn{1}{|c|}{ Palabra } & Frecuencia Total Misiones $y$ Visiones & $\%$ \\
\hline Clientes & 53 & $3.06 \%$ \\
\hline Desarrollo & 35 & $2.02 \%$ \\
\hline Financieras & 26 & $1.50 \%$ \\
\hline Servicios & 24 & $1.38 \%$ \\
\hline Mejor & 23 & $1.33 \%$ \\
\hline Nuestros & 18 & $1.04 \%$ \\
\hline Personas & 18 & $1.04 \%$ \\
\hline Líder & 15 & $0.87 \%$ \\
\hline Calidad & 14 & $0.81 \%$ \\
\hline Empresas & 14 & $0.81 \%$ \\
\hline Productos & 14 & $0.81 \%$ \\
\hline Accionistas & 13 & $0.75 \%$ \\
\hline Experiencia & 12 & $0.69 \%$ \\
\hline Soluciones & 12 & $0.69 \%$ \\
\hline Contribuir & 11 & $0.63 \%$ \\
\hline Valor & 11 & $0.63 \%$ \\
\hline País & 10 & $0.58 \%$ \\
\hline
\end{tabular}


Tabla 3. Tabla de frecuencias de las misiones de los bancos.

\begin{tabular}{|l|c|c|}
\hline \multicolumn{1}{|c|}{ Palabra } & Frecuencia Misiones & $\%$ \\
\hline Desarrollo & 26 & $3.37 \%$ \\
\hline Clientes & 19 & $2.47 \%$ \\
\hline Personas & 13 & $1.69 \%$ \\
\hline Servicios & 13 & $1.69 \%$ \\
\hline Calidad & 10 & $1.30 \%$ \\
\hline Contribuir & 10 & $1.30 \%$ \\
\hline Empresas & 10 & $1.30 \%$ \\
\hline Mejor & 10 & $1.30 \%$ \\
\hline
\end{tabular}

Tabla 4. Frecuencias de las visiones de la banca iberoamericana.

\begin{tabular}{|l|c|c|}
\hline Palabra & Total & $\%$ \\
\hline Clientes & 34 & $3.48 \%$ \\
\hline Líder & 25 & $2.56 \%$ \\
\hline Financieros & 22 & $2.25 \%$ \\
\hline Cliente & 12 & $1.23 \%$ \\
\hline Servicios & 11 & $1.13 \%$ \\
\hline Experiencia & 10 & $1.02 \%$ \\
\hline
\end{tabular}

Así mismo, se utiliza la herramienta de nube de palabras, en al análisis en conjunto de las misiones y de las visiones, en la Figura 1, se observa el resultado. Utilizando la herramienta de nube de palabras, para el análisis de contenido de las misiones en la Figura 2, se presentan resultados para los bancos estudiados. Finalmente, utilizando la herramienta de nube de palabras, para el análisis de contenido de las visiones en la Figura 3, se presentan resultados para los bancos estudiados.

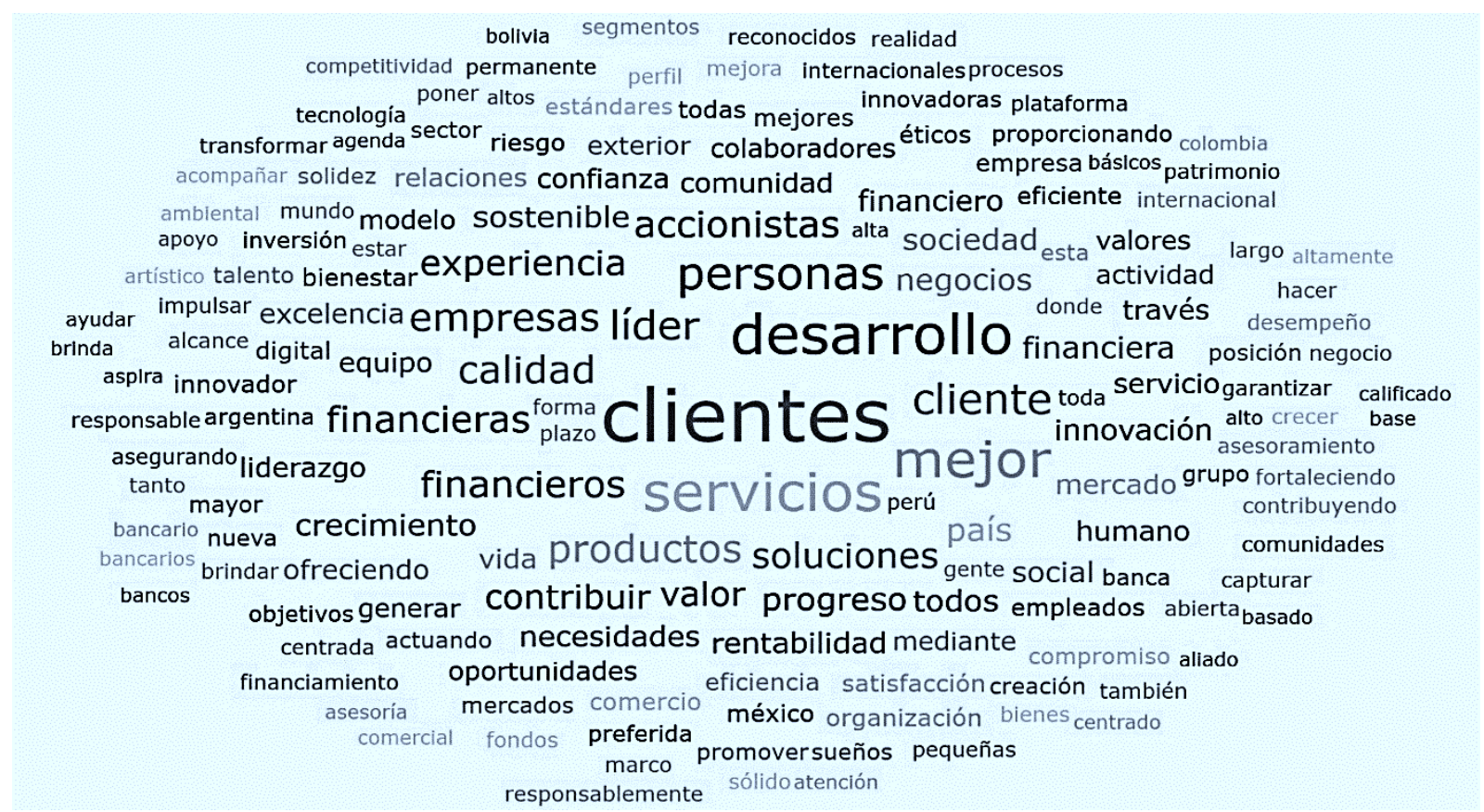

Fig. 1. Nube de palabras de las misiones y visiones unificadas de bancos iberoamericanos. 


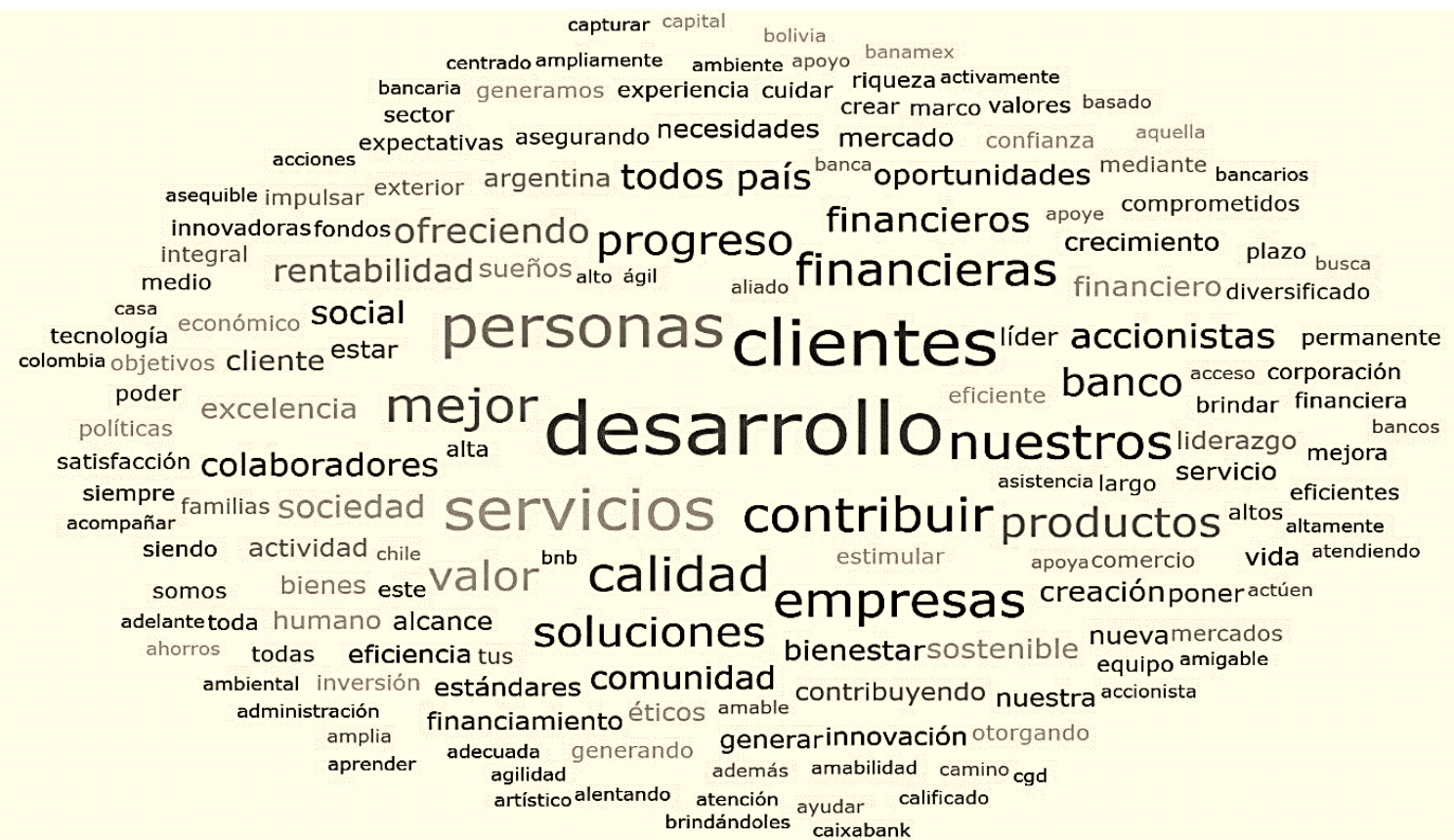

Fig. 2. Nube de palabras de misiones de los bancos iberoamericanos.

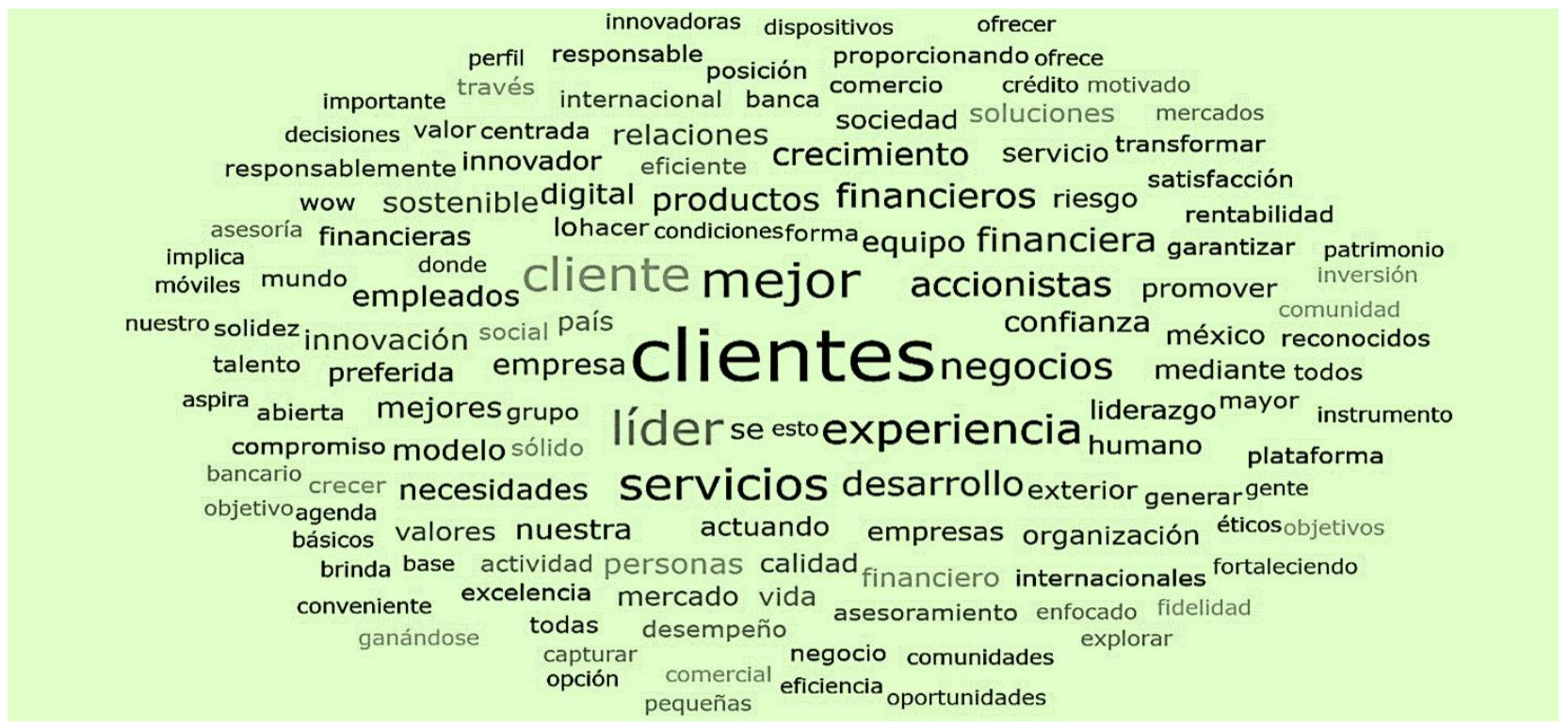

Fig. 3. Nube de palabras de visiones de los bancos iberoamericanos.

\section{Resultados por tipo de propiedad}

Este apartado se busca hacer una distinción entre la banca pública y privada en Iberoamérica. En primer lugar, en la Tabla 5, se puede apreciar la frecuencia en las misiones y visiones de las instituciones financieras privadas. Por otra parte, en la Tabla 6, se pueden aprecias las frecuencias de las instituciones financieras públicas en lberoamérica.

Tabla 5. Frecuencias de las misiones y visiones de la banca privada iberoamericana.

\begin{tabular}{|l|c|c|}
\hline \multicolumn{3}{|c|}{ Misiones Bancos Privados } \\
\hline Palabra & Frecuencia & $\%$ \\
\hline Desarrollo & 16 & 3.16 \\
\hline Clientes & 11 & 2.18 \\
\hline Personas & 11 & 2.18 \\
\hline Líder & 10 & 1.98 \\
\hline Empresa & 7 & 1.39 \\
\hline
\end{tabular}


Tablas 5 (continuación)

\begin{tabular}{|l|c|c|}
\hline \multicolumn{3}{|c|}{ Visiones Bancos Privados } \\
\hline Palabra & Frecuencia & $\%$ \\
\hline Clientes & 25 & 3.57 \\
\hline Líder & 18 & 2.57 \\
\hline Accionistas & 7 & 1.00 \\
\hline Servicios & 7 & 1.00 \\
\hline Experiencia & 6 & 0.86 \\
\hline
\end{tabular}

Tabla 6. Frecuencias de las misiones y visiones de la banca pública en iberoamericana.

\begin{tabular}{|l|c|c|}
\hline \multicolumn{3}{|c|}{ Misiones Bancos Públicos } \\
\hline Palabra & Frecuencia & $\%$ \\
\hline Desarrollo & 8 & 3.13 \\
\hline Servicios & 6 & 2.34 \\
\hline Calidad & 4 & 1.56 \\
\hline Empresas & 4 & 1.56 \\
\hline País & 4 & 1.56 \\
\hline Visiones Bancos Públicos \\
\hline Palabra & Frecuencia & $\%$ \\
\hline Exterior & 9 & 2.12 \\
\hline Negocios & 6 & 1.46 \\
\hline Desarrollo & 5 & 1.18 \\
\hline Clientes & 5 & 1.18 \\
\hline Servicios & 4 & 0.94 \\
\hline
\end{tabular}

\section{DISCUSIÓN}

Después de haber procesado 78 declaraciones de Misiones y Visiones, este estudio tenía como objetivo reconocer cuales eran las prioridades de la banca en Iberoamérica. Para realizar este análisis se toma en cuenta la información obtenida en las páginas web de los diferentes sitios, junto a otra información relevante y actualizada, como reportes, informes, códigos de buen gobierno, entre otros. De esta forma, se lograron realizar las tablas y gráficas por medio de Atlas T.I. Esta investigación revela la prioridad que los bancos le dan al desarrollo económico de los países donde se encuentra y su enfoque total hacía los clientes, donde centran todos sus procesos. Además, revela la importancia que se le da a los diferentes grupos de interés como clientes, sociedad, colaboradores, accionistas, entre los principales más destacados.

Asimismo, se muestra un comparativo entre la banca privada y pública, contraría a los resultados de (Ahmed, 2010) la banca iberoamericana es rica en cuanto a las declaraciones de este tipo de estrategias donde la gran mayoría la tiene siempre presente. Además, muestran el interés en el desarrollo de las regiones donde se encuentran y por brindar servicios de calidad a sus clientes. La diferencia entre tipo de institución privada o pública radica en el enfoque de la banca privada en los clientes y en la implementación de servicios de calidad, además, así como también un componente de mayor competitiva buscado ser líderes del marcado. Por otra parte, los dos tipos de instituciones bancarias tienen un enfoque en el desarrollo de las regiones donde se encuentran, sin embargo, las instituciones públicas tienen un mayor enfoque en negocios internacionales y se centra menos en la competitividad (Ahmed, 2010).

A diferencia de Palatonova et al (2018) donde al estudiar las misiones y visiones de los Bancos Islámicos se encontró una relación positiva entre la responsabilidad social empresarial desde las declaraciones de las misiones y visiones, en esta investigación, no hay una marcada relación, pero si entre las palabras que más se repiten se encuentran algunas relacionadas con esta estrategia, como son las palabras, clientes o desarrollo. También se evidencian diferencias con estudios en Malasia como Tan et al. (2017), donde se identifica desde las declaraciones de misión y visión la motivación para ser una banca sostenible, característica que no se evidencia en el estudio para lberoamérica.

Los hallazgos de esta investigación, se parecen más a los presentados en Kontolaimou et al. (2006), donde se analizaron las misiones de bancos del sudeste de Europa, y se identifica una mayor orientación hacia el cliente, y un menor énfasis en la sociedad o en los valores, también se identifica similitud con resultados de investigaciones sobre bancos de estados unidos, donde Bettinger (1985), encuentra en las declaraciones de 
las misiones, una orientación hacia el propósito de los bancos con relación a los clientes. Con relación a estudios globales como el propuesto por Bartkus et al. (2006), donde se identifica una relación entre la calidad de la declaración de la misión de los bancos, basada en las partes interesadas, los componentes y el cumplimiento de los objetivos, y el desempeño financiero; al contrastarlo con la presente investigación, es posible plantear que los Bancos Iberoamericanos estudiados, que son los más representativos de la región, incluyen en sus declaraciones palabras relacionadas con los mismos tres aspectos: las partes interesadas, los componentes y el cumplimiento de los objetivos.

En primer lugar, se destacaron los clientes, con una frecuencia en todas las Misiones y Visiones con un $\mathrm{f}=53$, esto sin contar que había palabras que se podían relacionar como personas o sociedad. Lo anterior, sin duda va en concordancia con los expuesto en la revisión teórica puesto que los bancos quieren innovar con productos y servicios que sean acordes a las necesidades de estos (Cotta, 2011). Además, la palabra desarrollo que fue la segunda que más frecuencia tenía con 35 , sin lugar a duda va encaminado a la finalidad o la función por la cual un banco surge que es la de traer progreso y riqueza a las naciones, en concordancia con lo propuesto por Fang et al. (2014). Por una parte, se puede ver que, en las nubes de palabras por tipo de propiedad, los bancos estatales se enfocan más en el desarrollo, ya que al ser públicos es casi su obligación contribuir con el progreso de este. Por otra parte, los privados, ya sean extranjeros o nacionales, se enfocan o resaltan en sus Misiones y Visiones el enfoque hacía el cliente, como también el deseo de ser mejores frente a la competencia ofreciendo productos y servicios, muy en la línea de investigaciones como las de Ahmed et al. (2017). Agregando a lo anterior, palabras como calidad, experiencias, soluciones son las formas en que estos pretender lograr su cometido.

Algunas de las limitaciones que se lograron observar al llevar a cabo la investigación, es la poca literatura que analiza estos dos aspectos de Misión y Visión en organizaciones e industrias tan importantes como lo es el sector bancario. Además, este tipo de investigaciones no permite ver que va más allá, puesto que los objetivos a los que apuntan los bancos como la transformación hacia lo digital no se ve tan reflejado en sus declaraciones de Misión y Visión. Sin embargo, aunque si hay frecuencias que hablan de sostenibilidad se pudo observar que hay muy pocas que hablen de este tema o algo relacionado con el medio ambiente.

Este estudio, es pertinente puesto que permite entender cuáles son los objetivos de los bancos dada su importancia en la economía mundial. Es importante que el lector entienda que la confianza de los clientes para que estos adquieran productos financieros que pueden contribuir al desarrollo y crecimiento no solo de la economía, sino también de la sociedad en general. Es por esto, que los bancos centran todos sus esfuerzos en satisfacer las necesidades del cliente para que estos confíen en estos y estos puedan seguir impulsando el progreso. De esta forma, se destaca la accesibilidad de la información obtenida, puesto que esta es pública a todas las personas y no solamente para ver aspectos como el de la Misión y Visión, se pueden ver desde los informes de gobierno corporativo, los informes anuales, los reportes de responsabilidad social, actas, balances, entre otros documentos.

\section{CONCLUSIONES}

A partir de los resultados obtenidos en la investigación y la discusión propuesta, se puede concluir lo siguiente: 1) La banca en Iberoamérica tiene dentro de sus prioridades, vistos a través de las misiones y visiones organizacionales, el desarrollo económico dentro de su enfoque corporativo, centrado en las necesidades de sus clientes en la banca personal y corporativa; 2) La Banca Iberoamericana evidencia una orientación estratégica a través de los grupos de interés, como la sociedad, los clientes, los colaboradores, los proveedores y los accionistas; 3) El desarrollo económico y el progreso son aspectos que se hacen muy evidentes en la banca pública, mientras que en la banca privada existe una mayor orientación hacia el desarrollo de nuevos productos y servicios tanto para clientes nuevos como para los antiguos; 4) no es claramente evidenciado en la misiones y visiones corporativas de los bancos en lberoamérica, la preocupación por el concepto de sostenibilidad, aunque se evidencia el interés por lo social, lo económico, lo ambiental está ausente de la premisas de las misiones y visiones bancarias.

\section{REFERENCIAS}

Ahmed, I., Aspirations of an Islamic bank: an exploration from stakeholders' perspective. https://doi.org/10.1108/IMEFM10-2014-0104, International Journal of Islamic and Middle Eastern Finance and Management, 9(1), 24-45 (2016).

Ahmed, I., Nawaz, M. M., y otros tres autores, Objectives of Islamic banks: a missive from mission statements and stakeholders' perceptions. https://doi.org/10.1108/JIABR-08-2014-0028, Journal of Islamic Accounting and Business Research, 8(3), 284-303 (2017).

Ahmed, A., Analysis of the Mission Statement of the Commercial Banks of Bangladesh. http://dx.doi.org/10.2139/ssrn.1633225, SSRN (2010). 
Allen, K. A., Kern, M. L., Vella-Brodrick, D., y Waters, L., Understanding the Priorities of Australian Secondary Schools Through an Analysis of Their Mission and Vision Statements. https://doi.org/10.1177/0013161X18758655, Educational Administration Quarterly, 54(2), 249-274 (2018).

Bakoğlu, R., y Aşkun, B., Mission statements of socially responsible firms: a content analysis, Journal of Global Strategic Management, 1(1), 66-74 (2007).

Bartkus, B., Glassman, M., y McAfee, B., Mission statement quality and financial performance, European Management Journal, 24(1), 86-94 (2006).

Billington, P., Analysis of mission and vision statements, Proceedings - Annual Meeting of the Decision Sciences Institute. 1, 398 (1996).

Braun, S., Wesche, J.S., y otros tres autores, Effectiveness of mission statements in organizations - A review. https://doi.org/10.5172/jmo.2012.18.4.430, Journal of Management and Organization, 18(4), 430-444 (2012).

Brickson, S.L., Organizational identity orientation: The genesis of the role of the firm and distinct forms of social value. https://doi.org/10.2307/20159339, Academy of Management Review, 32(3), 864-888 (2007).

Caplen, E., Top 1000 World Banks 2020, The Banker (2020).

Casilda, R., La banca española en Iberoamérica: Inversiones y perspectivas, Esic Market, (104), 9-32 (1999).

Cotta, P., Our mission and our values': An approach to Russian banks' communication strategies, Linguistic Insights, 134, 235-252 (2011).

Dhananjay, B., y Chandra, S., The electronic banking revolution in India, The Journal of Internet Banking and Commerce, 20(2), 110 (2015).

Fang, Y., Hasan, I., y Marton, K., Institutional development and bank stability: Evidence from transition countries. https://doi.org/10.1016/j.jbankfin.2013.11.003, Journal of Banking and Finance, 39(1), 160-176 (2014).

Freeman, R.E., Harrison, J.S., y otros tres autores, Stakeholder theory: The state of the art. Cambridge University Press Cambridge, Reino Unido (2010).

Ingenhoff, D., y Fuhrer, T., Positioning and differentiation by using brand personality attributes: Do mission and vision statements contribute to building a unique corporate identity?. https://doi.org/10.1108/13563281011016859, Corporate Communications, 15(1), 83-101 (2010).

Kontolaimou, A., Psallidas, D., y Pseiridis, A., An exploratory investigation of mission statements of banks in South Eastern European countries: customers vs. society?, International Journal of Financial Services Management, 2(1/2), 82-89 (2006).

Lacourt, C., y Musalem, M., Ranking 2020 Mayores 250 bancos un 2019 con Balance Positivo, Revista América Economía (2020).

Melewar, T.C., Determinants of the corporate identity construct: $A$ review of the literature. https://doi.org/10.1080/1352726032000119161, Journal of Marketing Communications, 9(4), 195-220 (2003).

Platonova, E., Asutay, M., Dixon, R., y Mohammad, S., The Impact of Corporate Social Responsibility Disclosure on Financial Performance: Evidence from the GCC Islamic Banking Sector. https://doi.org/10.1007/s10551-016-3229-0, Journal of Business Ethics, 151(2), 451-471 (2018).

Schure, P., Wagenvoort, R., y O'Brien, D., The efficiency and the conduct of European banks: Developments after 1992. https://doi.org/10.1016/j.rfe.2004.01.001, Review of Financial Economics, 13(4), 371-396 (2004).

Segura, E. A., y Valls, M. del C., Análisis estratégico de la banca ética en España a través de Triodos Bank. Financiación de proyectos sociales y medioambientales. https://doi.org/10.7203/CIRIEC-E.92.10805, Revista de Economía Publica, Social y Cooperativa, 92(1), 87-120 (2018).

Tan, L. H., Chew, B. C., y Hamid, S. R., A holistic perspective on sustainable banking operating system drivers. https://doi.org/10.1108/QRFM-12-2016-0052, Qualitative Research in Financial Markets, 9(3), 240-262 (2017).

Ting, H.-I., Financial development, role of government, and bank profitability: evidence from the 2008 financial crisis. https://doi.org/10.1007/s12197-016-9356-8, Journal of Economics and Finance, 41 (2), 370-391 (2017).

Vaismoradi, M., Turunen, H., y Bondas, T., Content analysis and thematic analysis: Implications for conducting a qualitative descriptive study. https://doi.org/ 10.1111/nhs.12048, Nursing and Health Sciences, 15(3), 398-405 (2013).

Weber, S., Origins of the European bank for reconstruction and development, International Organization, 48(1), 1-38 (1994). 